10. Wang H, Castiglioni C, Kacar Bayram A, Fattori F, Pekuz S, Araneda D, et al. Insights from genotype-phenotype correlations by novel SPEG mutations causing centronuclear myopathy. Neuromuscul Disord 2017; 27:836-842.

11. Wang H, Schanzer A, Kampschulte B, Daimaguler HS, Logeswaran T, Schlierbach H, et al. A novel SPEG mutation causes non-compaction cardiomyopathy and neuropathy in a floppy infant with centronuclear myopathy. Acta Neuropathol Commun 2018;6:83.

12. Lornage X, Sabouraud P, Lannes B, Gaillard D, Schneider R, Deleuze JF, et al. Novel SPEG mutations in congenital myopathy without centralized nuclei. J Neuromuscul Dis 2018;5:257-260.

13. Herkert JC, Abbott KM, Birnie E, Meems-Veldhuis MT, Boven LG, Benjamins M, et al. Toward an effective exome-based genetic testing strategy in pediatric dilated cardiomyopathy. Genet Med 2018. doi: $10.1038 /$ gim.2018.9
14. Teer JK, Green ED, Mullikin JC, Biesecker LG. VarSifter: visualizing and analyzing exome-scale sequence variation data on a desktop computer. Bioinformatics 2012;28:599-600.

15. Dubowitz V, Sewry CA, Oldfors A. Muscle biopsy: a practical approach. Ansterdam: Elsevier; 2013.

16. Schumann G, Bonora R, Ceriotti F, Clerc-Renaud P, Ferrero CA, Ferard G, et al. IFCC primary reference procedures for the measurement of catalytic activity concentrations of enzymes at 37 degrees C. Part 2. Reference procedure for the measurement of catalytic concentration of creatine kinase. Clin Chem Lab Med 2002;40:635-642.

17. Hsieh CM, Fukumoto S, Layne MD, Maemura K, Charles H, Patel A, et al. Striated muscle preferentially expressed genes alpha and beta are two serine/threonine protein kinases derived from the same gene as the aortic preferentially expressed gene-1. J Biol Chem 2000;275: 36966-36973.

\title{
INCREASED RISK OF MELANOMA IN C9ORF72 REPEAT EXPANSION CARRIERS: A CASE-CONTROL STUDY
} \author{
ISABEL SANTANA, MD, PHD ${ }^{1,2}$ \\ ${ }^{1}$ CHUC, Serviço de Neurologia, Praceta Prof. Mota Pinto, 3000-075, Coimbra, Portugal \\ ${ }^{2}$ Faculty of Medicine, University of Coimbra, Portugal \\ ${ }^{3}$ Centre for Neuroscience and Cell Biology, University of Coimbra, Portugal \\ ${ }^{4}$ Dermatology Department, Centro Hospitalar e Universitário de Coimbra, Portugal \\ Accepted 13 November 2018
}

MIGUEL TÁBUAS-PEREIRA, MD iD ${ }^{1,2 *}$ LUCIANO ALMENDRA, MD, ${ }^{1 *}$ MARIA ROSÁRIO ALMEIDA, MSC, PHD, ${ }^{3}$ JOÃO DURÃES, MD, ${ }^{1}$ ANDRÉ PINHO, MD, ${ }^{4}$ ANABELA MATOS, MD, ${ }^{1}$ LUIS NEGRÃO, MD, ${ }^{1}$ ARGEMIRO GERALDO, MD, ${ }^{1}$ and

ABSTRACT: Introduction: Amyotrophic lateral sclerosis (ALS) and frontotemporal lobar degeneration (FTLD) are considered part of the same pathological spectrum. There is an increased risk of ALS in patients who have had melanoma. The risk of FTLD in melanoma (or cancer) patients is unknown. We aimed to study if C9ORF72 expansion is linked to a higher prevalence of melanoma. Methods: We selected patients with a diagnosis in the ALS-FTLD spectrum who were tested for pathogenic mutations. Medical history was reviewed, to identify those with pathologically documented melanomas. Results: We included 189 patients. Sixty-two had identified pathogenic mutations (39 C9ORF72). C9ORF72 carriers had a significantly higher risk of melanoma (odds ratio $=24.709 ; P<0.007$ ). There was no association with phenotype. Conclusions: These findings suggest that patients with a history of melanoma may have an increased probability of carrying a C9ORF72 repeat expansion. ALS or FTLD carriers of C9ORF72 should undergo surveillance for skin changes.

Muscle Nerve 59:362-364, 2019

Several studies have examined the risk of cancer in neurodegenerative diseases, including Parkinson and Alzheimer diseases, which have been reported to have a protective effect against the overall development of

Abbreviations: ALS, amyotrophic lateral sclerosis; C9ORF72, chromosome 8 opening read frame 72; CHMP2B, charged multivesicular body protein 2B; FTLD, frontotemporal lobar degeneration; FUS, fused-in-sarcoma; GRN, progranulin; MAPT, microtubule-associated protein tau; SOD, superoxide dismutase; TBK1, TANK-binding kinase 1; VCP, valosincontaining protein.

Key words: amyotrophic lateral sclerosis, C9ORF72, frontotemporal dementia, melanoma, skin

Funding: Nothing to report

Conflicts of Interest: None of the authors has any conflict of interest to disclose.

*These authors contributed equally to this study.

Correspondence to: M. Tábuas-Pereira; e-mail: miguelatcp@gmail.com

(C) 2018 Wiley Periodicals, Inc

Published online 17 November 2018 in Wiley Online Library

(wileyonlinelibrary.com). DOI 10.1002/mus.26383 cancer. $^{1-5}$ The same overall protective effect has been reported recently in amyotrophic lateral sclerosis (ALS) ${ }^{6}$ but some types of cancer seem to have increased prevalence in this population. There is mounting evidence of an increased risk of ALS in patients who have had melanoma., ${ }^{7,8}$ ALS and frontotemporal lobar degeneration (FTLD) are considered part of the same pathological spectrum and share common pathogenic genetic mutations, with the chromosome 9 open reading frame 72 (C9ORF72) expansion being the most common in both. ${ }^{9}$ A small study in FTLD reports overall lower risk of cancer. ${ }^{10}$

Several genes have been implicated in the association between cancer and neurodegenerative diseases, including progranulin $(\mathrm{GRN}),{ }^{11}$ fused-in-sarcoma (FUS), ${ }^{12}$ and SQSTM1. ${ }^{13}$ However, we still lack the understanding of the underlying mechanism that would explain such association. ${ }^{14}$ Exploring the relationship between cancer and neurodegenerative diseases could provide new clues relevant to the etiologies and potential therapies for both sets of conditions.

Of interest, analysis of skin biopsies of $C 9 O R F 72$ repeat expansion ALS patients revealed early TDP-43 deposition and abnormal extracellular matrix findings, ${ }^{15}$ suggesting a possible link between this expansion and skin disease.

We aimed to study the hypothesis that C9ORF72 expansion is linked to a higher prevalence of melanoma.

\section{PATIENTS AND METHODS}

Patients. We selected ALS-FTLD spectrum patients, followed at the dementia and neuromuscular clinics of our 
department with the most recent appointment between 2010 and 2017, who were tested for pathogenic mutations in superoxide dismutase (SOD), TANK-binding kinase 1 (TBK1), GRN, microtubule-associated protein tau (MAPT), valosincontaining protein (VCP), FUS, charged multivesicular body protein 2B (CHMP2B), or C9ORF72 repeat expansions.

This study was approved by our hospital ethical committee, and all patients or their close relatives gave consent for data collection.

Methods. We reviewed all subjects' medical history to identify those with histopathologically documented melanoma during their lifetime. This was done with the families (and the patients whenever they could give reliable information) and by reviewing all the patients' medical records.

To investigate a possible link between c90rf72 repeat expansion and melanoma, we compared the risk of melanoma between C9ORF72 repeat expansion carriers and all patients who tested negative for C9ORF72 expansion. We further compared the risk of melanoma between C9ORF72 carriers and patients with other identified pathogenic mutations.

We also compared melanoma prevalence across different phenotypes and basal cell carcinoma prevalence between C9ORF72 carriers and noncarriers.

Statistical Analysis. Statistical analysis was performed using SPSS statistical software (version 20.0; SPSS Inc., Chicago, IL). Categorical data are presented as frequency (percentage) and were compared using $\chi^{2}$-test. Continuous variables were compared using Student's $t$-test. To estimate the association between carrying a C9ORF72 expansion and developing melanoma, a logistic regression was performed to calculate the odds ratio, adjusted for age. A Bejamini-Hochberg post hoc correction for multiple comparisons was performed, giving a $\alpha=0.0083$ ).

\section{RESULTS}

We included 189 patients. Of those, 157 patients had a primary FTLD diagnosis and 32 an ALS diagnosis. Of the total, 62 had identified pathogenic mutations: 39 subjects had C9ORF72 repeat expansions, 3 had MAPT mutations, and 20 had GRN mutations. Patient diagnosis and mutation status are provided in Table 1.

The mean age of all subjects was of $63.8( \pm 10.5)$ years. Mutation carriers were significantly younger $(59.3 \pm 10.4$ years; $P<0.001)$, as were the C9ORF72 expansion carriers $(60.6 \pm 10.7$ years old; $P=0.031)$. $54.5 \%$ of the population was male, and there were no gender differences between groups.

Seven patients had a diagnosis of melanoma; 6 of them were carriers of a C9ORF72 repeat expansion, and the other had no identified mutation. Three additional patients had basal cell carcinoma: 2 of them were C9ORF72 expansion carriers and 1 had no identified pathogenic mutation. All the 6 patients with a $C 9 O R F 72$ expansion and melanoma ( $15.4 \%$ of the C9ORF72 carriers) belonged to 4 unrelated families. Five of them had ALS and one had FTLD. Three of them were from the same family ( 7 members carrying the mutation and manifesting disease, 3 of whom had melanoma). The patient with melanoma without a pathogenic mutation was unrelated to any of the patients with melanoma.
Table 1. Diagnosis and mutation status for all patients (white squares) and only patients with melanoma (black-filled squares).

\begin{tabular}{lrrrc}
\hline & ALS & FTLD & Total & Melanoma \\
\hline C9ORF72 & 14 & 25 & 39 & 6 \\
PGRN & 0 & 20 & 20 & 0 \\
MAPT & 0 & 3 & 3 & 0 \\
No mutation & 18 & 109 & 127 & 1 \\
Total & 32 & 157 & 189 & 7 \\
Melanoma & 5 & 2 & 7 & \\
\hline
\end{tabular}

As depicted in FIGURE 1, C9ORF72 carriers had a significantly higher prevalence of melanoma $(P<0.001)$, comparing with all the other patients with a negative test for this expansion (odds ratio $=24.709$; 95\% confidence interval, 2.440-249.804; $P<0.007$; Nagelkerke R square of $\left.0.259 ; \chi^{2}=10.637\right)$. Compared only with other mutation carriers, melanoma prevalence was still significantly higher $(P=0.048)$. There was association with neither FTLD nor ALS $(P=0.216)$ in $C 9 O R F 72$ carriers.

Other melanoma-causing mutations were excluded in the two living C9ORF72 carriers with history of melanoma. CDKN2A was tested in the 2 patients with melanoma and C9ORF72 repeat expansions and were negative. One of the patients was further studied for $C D K 4, M C 1 R, C D K N 2 B$, and GNAQ and was negative for pathogenic mutations in those genes.

\section{DISCUSSION}

Our study suggests a link between $C 9 O R F 72$ repeat expansions and increased risk of melanoma. Additionally, melanoma's prevalence in C9ORF72 carriers is higher than that of basal cell carcinoma, which is the inverse of the general population. ${ }^{16}$ Several previous studies analyzing the risk of ALS in cancer patients revealed a link between both conditions. None of these studies analyzed the role of specific mutations in this association. We speculate that the increased risk of melanoma found in those studies can be (at least partially) due to the existence of occult $C 9 O R F 72$ repeat expansion carriers on those samples. It could also be that a common pathway is involved in the ALS/FTLD spectrum and melanoma development, in which C9ORF72 repeat expansions may play a role.

It has been shown that TDP-43 nuclear overexpression with no cytoplasmic inclusions can be detected in skin biopsies from ALS patients. ${ }^{15}$ In addition, analysis of cultured fibroblasts and keratinocytes from skin biopsies of C9ORF72 repeat expansion ALS patients reveals abnormal extracellular matrix findings, such as epidermal undifferentiation, abnormal dermoepidermal junction, delamination keratinocyte infiltration, and collagen disorganization. ${ }^{17}$ Consistently, an in silico bioinformatics data mining analysis of the molecular network of C9ORF72 showed a down-regulation of collagen-related genes as well as genes implicated in the assembly, synthesis, and cross-linking of collagen 


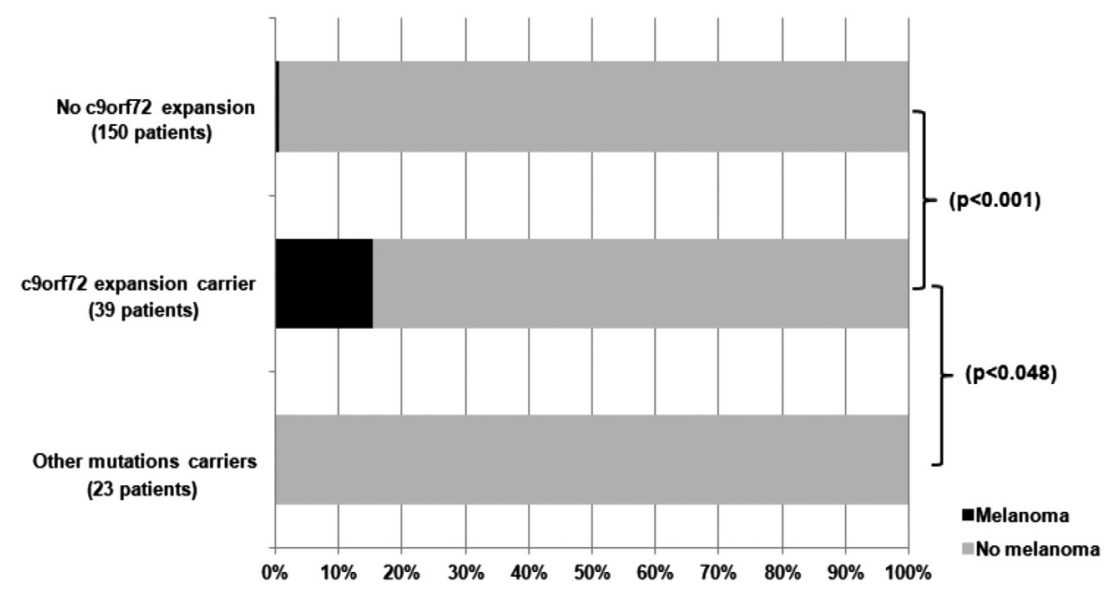

FIGURE 1. Comparison of melanoma prevalence among patients without a C9ORF72 expansion (including patients with other mutations) in the first line ( 1 case in 150 patients, $0.7 \%$ ) with C9ORF72 expansion carriers in the second line (6 cases in 39 patients, $15.4 \%)$ and other pathogenic mutation carriers ( 0 cases in 23 patients, $0.0 \%$ ).

fibrils. ${ }^{18}$ These findings suggest that cytoskeletal dynamics and/or remodeling of extracellular matrix disturbances might be key factors of the disease and associate C9ORF72 repeat expansions with those changes, possibly increasing the risk for melanoma.

One possible bias would be genetic linkage of the C9ORF72 repeat expansion to a mutation in a nearby gene involved in melanoma development. Several gene mutations have been associated with melanoma, namely CDK4, CDKN2A, GNAQ, RB1, PPP6C, RAC1, SNX31, TACC1, STK19, ARID2, BRAF, NRAS, HRAS, TERT, KIT, TP53, EGFR, ERBB4, PIK3CA, PDGFRA ${ }^{19}$ Some of these $(C D K N 2 A$ and $G N A Q)$ localize in the short arm of the chromosome 9. CDKN2A was tested in the 2 patients with melanoma and $C 9 O R F 72$ repeat expansions and was negative. One of the patients was further studied for $C D K 4, M C 1 R, C D K N 2 B$, and GNAQ somatic mutations and was negative for pathogenic mutations in those genes. A phenomenon of epistasis between $\mathrm{C} 90 \mathrm{RF} 72$ and $C D K N 2 A$ or $G N A Q$ is also possible, given their relative proximity, and should be further addressed.

The major limitation of this work is the low number of melanomas, with half of them coming from the same family. There may be an underlying mutation or environmental risk factor that we did not identify. However, even if we exclude those patients, the difference would still be significantly higher $(P=0.002)$. Also, there was a statistically significant difference in the ages of the groups. This was expected given the lower age of onset of patients with pathogenic mutations. However, this difference is only 3 years, and if affecting the prevalence of melanoma, would only potentially increase the difference, as they had less time to develop melanoma.

These findings suggest that ALS or FTLD patients with a personal history of melanoma may be at increased risk of carrying a C9ORF72 repeat expansion, and that genetic testing for C9ORF72 might be considered in those patients. Carriers of C9ORF72 should undergo surveillance for skin changes.
Ethical Publication Statement: We confirm that we have read the Journal's position on issues involved in ethical publication and affirm that this report is consistent with those guidelines.

\section{REFERENCES}

1. Fois AF, Wotton CJ, Yeates D, Turner MR, Goldacre MJ. Cancer in patients with motor neuron disease, multiple sclerosis and Parkinson's disease: record linkage studies. J Neurol Neurosurg Psychiatry 2010;81:215-221.

2. Roe CM, Fitzpatrick AL, Xiong C, Sieh W, Kuller L, Miller JP, et al. Cancer linked to Alzheimer disease but not vascular dementia. Neurology 2010;74:106-112.

3. Inzelberg R, Jankovic J. Are Parkinson disease patients protected from some but not all cancers? Neurology 2007;69:1542-1550.

4. Driver JA, Beiser A, Au R, Kreger BE, Splansky GL, Kurth T, et al. Inverse association between cancer and Alzheimer's disease: results from the Framingham Heart Study. BMJ 2012;344:e1442

5. Kareus SA, Figueroa KP, Cannon-Albright LA, Pulst SM. Shared predispositions of parkinsonism and cancer: a population-based pedigreelinked study. Arch Neurol 2012;69:1572-1577.

6. Gibson SB, Abbott D, Farnham JM, Thai KK, McLean H, Figueroa KP et al. Population-based risks for cancer in patients with ALS. Neurology 2016;87:289-294.

7. Baade PD, Fritschi L, Freedman DM. Mortality due to amyotrophic lateral sclerosis and Parkinson's disease among melanoma patients. Neuroepidemiology 2007;28:16-20.

8. Freedman DM, Curtis RE, Daugherty SE, Goedert IJ, Kuncl RW, Tucker MA. The association between cancer and amyotrophic lateral sclerosis. Cancer Causes Control 2013;24:55-60.

9. Majounie E, Renton AE, Mok K, Dopper EG, Waite A, Rollinson S, et al. Frequency of the C9orf72 hexanucleotide repeat expansion in patients with amyotrophic lateral sclerosis and frontotemporal dementia: a cross-sectional study. Lancet Neurol 2012;11:323-330.

10. Katisko K, Haapasalo A, Koivisto A, Kruger J, Hartikainen P, Korhonen V, et al. Low prevalence of cancer in patients with frontotemporal lobar degeneration. J Alzheimers Dis 2018;62:789-794.

11. He Z, Ismail A, Kriazhev L, Sadvakassova G, Bateman A. Progranulin (PC-cell-derived growth factor/acrogranin) regulates invasion and cell survival. Cancer Res 2002;62:5590-5596.

12. Dormann D, Haass C. Fused in sarcoma (FUS): an oncogene goes awry in neurodegeneration. Mol Cell Neurosci 2013;56:475-486.

13. Thompson HG, Harris JW, Wold BJ, Lin F, Brody JP. p62 overexpression in breast tumors and regulation by prostate-derived Ets factor in breast cancer cells. Oncogene 2003;22:2322-2333.

14. Albert SM. Neurodegenerative disease and cancer: a critical role for melanoma? Neuroepidemiology 2010;35:305-306.

15. Suzuki M, Mikami H, Watanabe T, Yamano T, Yamazaki T, Nomura M, et al. Increased expression of TDP-43 in the skin of amyotrophic lateral sclerosis. Acta Neurol Scand 2010;122:367-372.

16. Wu S, Han J, Li WQ, Li T, Qureshi AA. Basal-cell carcinoma incidence and associated risk factors in U.S. women and men. Am J Epidemiol 2013;178:890-897.

17. Pare B, Touzel-Deschenes L, Lamontagne R, Lamarre MS, Scott FD, Khuong HT, et al. Early detection of structural abnormalities and 
cytoplasmic accumulation of TDP-43 in tissue-engineered skins derived from ALS patients. Acta Neuropathol Commun 2015;3:5.

18. Satoh J, Yamamoto Y, Kitano S, Takitani M, Asahina N, Kino Y. Molecular network analysis suggests a logical hypothesis for the pathological role of c9orf72 in amyotrophic lateral sclerosis/frontotemporal dementia. J Cent Nerv Syst Dis 2014;6:69-78.

19. Tsao H, Chin L, Garraway LA, Fisher DE. Melanoma: from mutations to medicine. Genes Dev 2012;26:1131-1155.

\title{
VCP MYOPATHY: A FAMILY WITH UNUSUAL CLINICAL MANIFESTATIONS
}

\author{
XUAN GUO, MS, ZHE ZHAO, MD, PhD, HONGRUI SHEN, MS, BING QI, MD, PhD, NAN LI, MS, and JING HU, MD, PhD \\ Department of Neuromuscular Disease, The Third Hospital of Hebei Medical University, 139\# Ziqiang Road, Shijiazhuang City, Hebei \\ Province, 050051, P. R. China \\ Accepted 23 November 2018
}

\begin{abstract}
Introduction: Valosin-containing protein (VCP) variants that affect muscle, bone, and the nervous system are termed multisystem proteinopathy. VCP myopathy is manifested as limb-girdle weakness, distal weakness and scapuloperoneal weakness. Methods: We reviewed clinical, genetic, and muscle biopsy data from 6 members of a family with VCP myopathy. Results: Clinical features of family members were complex and included dementia, myopathy, and hearing impairment. Ophthalmoplegia, ptosis, and dysphagia were present in 3 siblings. Rimmed vacuoles were observed in muscle biopsies, consistent with the pathological changes of VCP myopathy. A heterozygous $V C P \quad c .463 C>A$ (p.R155S) that segregated in an autosomaldominant pattern was identified by genetic analysis. Conclusions: VCP myopathy can cause unusual manifestations that include ophthalmoplegia, ptosis, and dysphagia. This study increased our understanding of the clinical manifestations of VCP myopathy.
\end{abstract}

Muscle Nerve 59:365-369, 2019

The gene valosin-containing protein (VCP) encodes the VCP protein comprising of 806 amino acids, located at 9p13.3. VCP is a member of the ATPases associated with various cellular activities (AAA+) superfamily ${ }^{1}$ and consists of an N-terminal domain, ATP enzyme domains (D1, D2), C-terminal domain and intermediate junction regions. ${ }^{2}$ VCP is a ubiquitously-expressed hexameric protein involved in various ubiquitin-dependent cellular processes and several essential cell protein pathways including cell membrane fusion, cell cycle, postmitotic Golgi reassembly, nuclear envelope reconstruction, suppression of apoptosis, DNA damage response, and the ubiquitin-proteasome degradation pathway. ${ }^{3-5}$ VCP disease is a rare, autosomal dominant (AD) disorder first reported in $1982^{6}$ that may present with

Additional supporting information may be found in the online version of this article.

Abbreviations: $A D$, autosomal dominant; $A L P$, alkaline phosphatase CMAP, compound muscle action potentials; CK, creatine kinases; EMG, electromyography; FTD, frontotemporal dementia; IBM, inclusion body myopathy; MRC, Medical Research Council; MoCA, Montreal Cognitive Assessment; NCS, nerve conduction studies; NGS, next generation sequencing; OPDM, oculopharyngodistal myopathy; PDB, Paget disease of bone; VCP, valosin-containing protein

Key words: distal weakness, dysphagia, ophthalmoplegia, ptosis, VCP myopathy

Conflicts of Interest: The authors declare no conflicts of interest.

Correspondence to: J. Hu; e-mail: jinghu5510@163.com

(C) 2018 Wiley Periodicals, Inc

Published online 29 November 2018 in Wiley Online Library

(wileyonlinelibrary.com). DOI 10.1002/mus.26389 varying combinations of inclusion body myopathy (IBM), Paget disease of bone (PDB), and frontotemporal dementia (FTD) (OMIM\#167320), or may manifest as amyotrophic lateral sclerosis, Parkinson's disease, cardiomyopathy, Charcot-Marie-Tooth disease, or hereditary spastic paraparesis. ${ }^{7}$

The most common clinical presentation of VCP disease is myopathy, seen in almost $90 \%$ of cases and characterized by late adult-onset shoulder and pelvic girdle weakness as well as distal myopathy. Facial muscles are occasionally involved. The serum creatine kinase (CK) is normal or slightly elevated. The most common findings of muscle biopsy are rimmed vacuoles and ubiquitin-, TDP-43-, and VCP-positive inclusions. ${ }^{8}$ PDB is seen in approximately $50 \%$ of patients, characterized by abnormal bone homeostasis resulting in polyostotic skeletal disorganization, pathological fractures, or osteolytic bone lesions, high alkaline phosphatase (ALP) and radiographic changes. FTD is recognized in one third of patients, presenting with early behavioral or language changes. Here, we report on a family of 6 VCP patients with a heterozygous missense mutation $V C P$ c.463C $>$ A (p.R155S) whose main clinical manifestation was myopathy with some unusual features.

\section{MATERIALS AND METHODS}

This study was approved by the Ethics Committee of the Third Hospital of Hebei University. Written informed consent was obtained from all patients and/or their legal guardians. Three siblings were clinically assessed, and their medical records including serum CK and ALP levels; radiographs of the pelvis, skull, and spine; lower limb muscle brain MRI; and needle electromyography (EMG) and nerve conduction studies (NCS) were reviewed. Information about the other patients was obtained from a description given by their guardians. Blood samples were collected from 9 family members.

Open muscle biopsy was performed on the tibialis anterior of the 3 siblings under local anesthesia. The specimens were stained with routine stains using standard techniques. In addition, ultrastructural examination was performed on the proband.

The target gene ( 1 patient)/whole-exome (4 patients) capture experiment was conducted following the manufacturer's protocol (My Genostics, Beijing, China) ${ }^{9}$ Confirmation of the findings of next generation sequencing (NGS) and segregation 